\title{
DLR-Hand II: Next Generation of a Dextrous Robot Hand
}

\author{
J. Butterfaß, M. Grebenstein, H. Liu and G. Hirzinger \\ German Aerospace Research Center (DLR) \\ Institute of Robotics and Mechatronics \\ P.O. Box 1116, D-82230 Wessling, Germany \\ Joerg.Butterfass@dlr.de
}

\begin{abstract}
- this paper outlines the 2nd generation of multisensory hand design at DLR. The results of the use of DLR's Hand I were analyzed and enabled - in addition to the big efforts made in grasping technology - to design the next generation of dextrous robot hands. An open skeleton structure for better maintenance with semi shell housings and the new automatically reconfigurable palm have been equipped with more powerful actuators to reach $30 N$ on the fingertip. Newly designed sensors as the 6 DOF fingertip force torque sensor and integrated electronics together with the new communication architecture which enables a reduction of the cabling to the hand to only 12 lines outline the electronics concept. The Cartesian impedance control of all the fingers completes the new hand with its 13 DOF to what it is: the next step to autonomous and humanoid grasping
\end{abstract}

\section{Introduction}

In 1997 DLR developed one of the first articulated hands with completely integrated actuators and electronics (fig. 1) [4]. This well known hand has been in

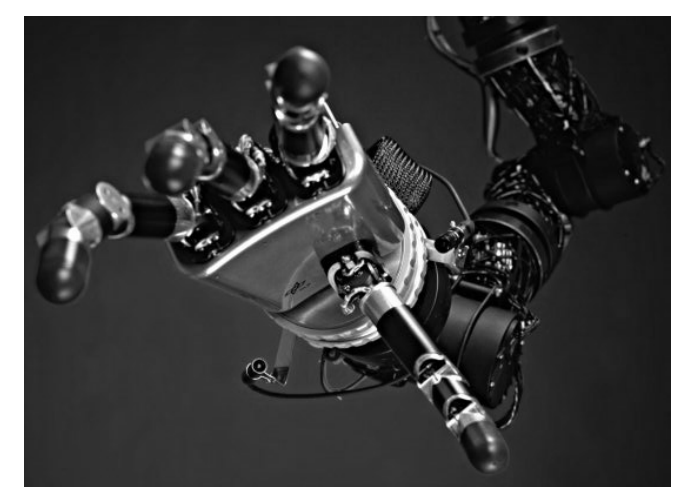

Figure 1. DLR's Hand I

use for several years and has been a very useful tool for research and development of grasping. The experiences with Hand I accumulated to a level that enabled us to design a new hand according to a fully integrated mechatronics concept which yields a reasonably better performance in grasping and manipulation and therefore accelerates further developments.

\section{Design Philosophy}

In order to achieve the goal of maximum flexibility and performance our philosophy is the miniaturization and complete integration of all components of the hand and also the massive reduction of cabling.

As on DLR's Hand I the main aspects in developing the new hand were maximum performance to improve autonomous grasping and fine manipulation possibilities and the use of fully integrated actuators and electronics without a forearm. This is the only possibility to use an articulated hand on different types of robots which are not specially prepared to be used with hands. Hands with forearms [5] or hands with just grasping abilities allow for a much smaller and thus more anthropomorphic design due to the possibility of using the additional space in the forearm for actuators and electronic components, but restrict the usability with e.g. industrial robots. Farther displacement of those components as known from the MIT-Utah Hand [3] nearly disables the use on mobile robots.

Furthermore the hand must be easy to maintain and use and even economically rebuild-able in case of any damage by daily research usage. For a good overview of more than fifty dextrous robot hands see [6].

\section{The DLR Hand II}

\subsection{Open Skeleton Design}

Due to maintenance problems with Hand I and in order to reduce weight and production costs the fingers and base joints of Hand II were realized as an open skeleton structure. The open structure is covered by 4 semi shells and one 2-component fingertip 
housing realized in stereolitography and vacuum mold (fig. 2). This enables us to test the influence of different shapes of the outer surfaces on grasping tasks without redesigning finger parts.

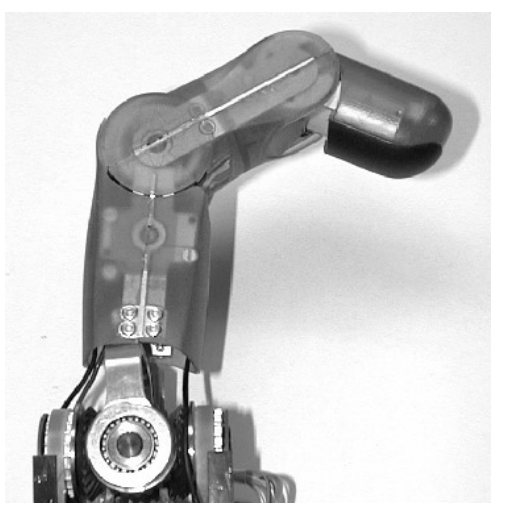

Figure 2. Finger with semi shell housings and rubber skin fingertip.

\subsection{Kinematic Design of DLR's Hand II}

The design process started on an anthropomophic base by evaluation of different workspace/manipulability measures like those of Salisbury [7] or Yoshikawa [9] to get optimal ratios of link lengths of one finger. The desired objects to be manipulated and technological restrictions resulted in absolute link lengths.

The second step was to get suitable hand kinematics. The main target developing Hand II beside the ability for fine manipulation has been the improvement of the grasping performance in case of precisionand power-grasp. Therefore the design of Hand II was

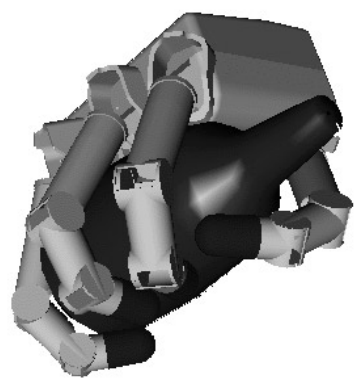

Figure 3. Optimization of kinematics with scalable handmodel.

based on performance tests with scalable virtual models as seen in fig. 3. Soon it turned out very impor- tant to be able to change the position of the 4th finger and the thumb as well. To perform power-grasps it is absolutely necessary to have a nearly parallel position of the second, third and forth finger as seen in fig. 4. On the other hand performing precision-grasps

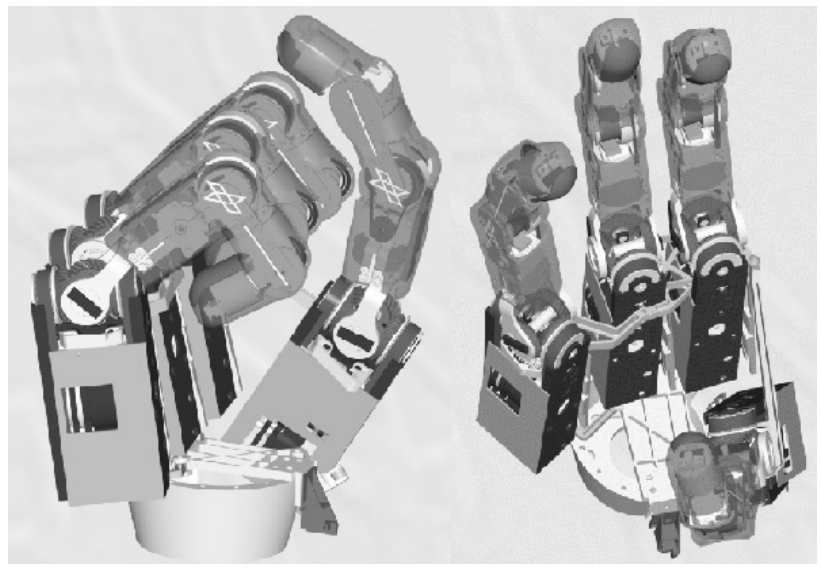

Figure 4. Simulation of Hand II in power-grasp- and fine manipulation configuration.

and fine-manipulation requires huge regions of intersection of the ranges of motion and the opposition of thumb and ring finger (fig. 4). Therefore Hand II was designed with an additional minor degree of freedom which enables to use the hand in 2 different configurations. This degree of freedom is a slow motion type to reduce weight and complexity of the system. The motion of the first and the fourth finger are both realized with just one brushed dc motor using a spindle gear.

The realized finger positions for both types of grasping were designed virtually and mapped to each other using the positions of 2 nd and 3rd finger. Realizable kinematics were calculated and imported to the two virtually found configurations and optimized unless the actual configuration with an overall number of 13 DOF was found (fig. 4).

\subsection{Actuator System}

The three independent joints (there is one additional coupled joint) of each finger are equipped with appropriate actuators. The actuation systems essentially consist of brushless dc-motors, tooth belts, harmonic drive gears and bevel gears in the base joint. The configuration differs between the different joints. The base joint with its two degrees of freedom is of differential bevel gear type, the harmonic drive gears for geometric reasons being directly coupled to the mo- 
tors. The differential type of joint (fig. 5) allows to use the full power of the two actuators for flexion or extension. Since this is the motion where most of the

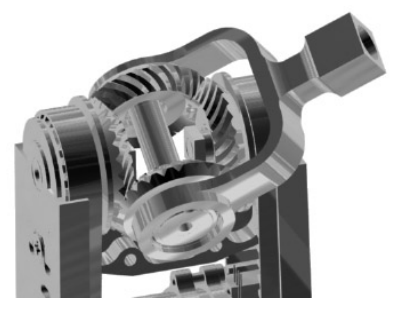

Figure 5. Differential bevel gear of the new basejoint.

available torque has to be applied, it allows to use the torque of both actuators jointly for most of the time. This means that we can utilize smaller motors.

The actuation system in the medial joint is designed to meet the conditions in the base joint when the finger is in stretched position and can apply a force of up to $30 \mathrm{~N}$ on the finger tip. Here the motor is linked to the gear by the transmission belt (see [8] for advantages). The motor in the medial joint has less power than the motors in the base joint, however there is an additional reduction of $2: 1$ by the transmission belt. Thus we achieve the torque which corresponds to the torque created by the two motors in the base joint for an external force of $30 \mathrm{~N}$ on the finger tip. The harmonic drives used are of the same type for all joints, since the smallest appropriate type can stand the torque for both types of actuation.

\subsection{Sensor Equipment}

A dextrous robot hand for teleoperation and autonomous operation needs (as a minimum) a set of force and position sensors. Various other sensors add to this basic scheme (see table). Each joint is equipped with strain gauge based joint torque sensors and specially designed potentiometers based on conductive plastic. Besides the torque sensors in each joint we designed a tiny six dimensional force torque sensor for each finger tip which will be explained more precisely in 3.5. The potentiometers, each with an analogous filter of third order, would not be absolutely necessary, since one may calculate the joint position from the motor position, however they provide us with a more accurate information of joint position, and they can by the way eliminate the necessity of referencing the fingers after power up. In case of not using the potentiometers one would have to consider the elasticity of the transmission belt and the harmonic drive. With the potentiometer we achieve a resolution for the joint angles of $1 / 10^{\circ}$, this means approximately 10 bits for the joint.

\begin{tabular}{|c|c|c|}
\hline sensor type & $\begin{array}{c}\text { count/ } \\
\text { finger }\end{array}$ & range (resolution) \\
\hline joint position & 3 & $\begin{array}{c}110^{\circ}(10 \mathrm{bit}) ; \\
120^{\circ}(10 \mathrm{bit})\end{array}$ \\
\hline joint torque & 3 & $2.4 ; 4.8 \mathrm{Nm}(11 \mathrm{bit})$ \\
\hline force/torque & 1 & $10-40 \mathrm{~N} ; 150 \mathrm{Nmm}(11 \mathrm{bit})$ \\
\hline motor speed & 3 & \\
\hline temperature & 6 & $0-125^{\circ} \mathrm{C}(8 \mathrm{bit})$ \\
\hline
\end{tabular}

Since the base joint is of differential type, one has to calculate the joint position of the base joint from the potentiometer values. There was no way to measure the joint position directly due to space restrictions. For increasing the controllability of the actuators we appreciate speed sensors. Like in DLR's first generation hand we utilize so called Tracking Converters [1]. In contrast to the old version the complete calculation is done by software since there is enough computing power available now. The sensor itself is basically a position sensor with very high resolution, where the speed can be calculated by differentiation of the position signal. Each motor is equipped with two linear Hall effect sensors which are used for commutation of the motors as well. These sensors supply two sinusoidal signals with a phase shift of $120^{\circ}$. The position within the magnetic cyle of the motor is calculated from these signals. By additionally counting the cycles the position can be calculated. This type of sensor gives us just a relative position of the motor and has thus to be referenced after power up.

\subsection{Force Torque Fingertip Sensor}

A tiny six dimensional force torque sensor $(20 \mathrm{~mm}$ in diameter and $16 \mathrm{~mm}$ in height) as shown in Fig. 7 with full digital output has been developed for the fingertip.

The force and torque measure ranges are $10 \mathrm{~N}$ for $F_{x}$ and $F_{y}, 40 \mathrm{~N}$ for $F_{z}, 150 \mathrm{Nmm}$ for $M_{x}, M_{y}$ and $M_{z}$ respectively. Also a $200 \%$ mechanical overload protection is provided in the structure.

Measuring principle: The mechanical structure (as shown in Fig. 6) of the sensor is composed of two sensitive parts, one is a round plate (base element) with three symmetrical sensitive beams, another is a cantilever beam.

There are three elastic beams in the base element, which are sensitive to the $M_{x}, M_{y}$ and $F_{z}$ by measuring strain $\epsilon_{1}, \epsilon_{2}$ and $\epsilon_{3}$. The cantilever beam is a rectangular pipe with a very thin wall. By using specialized torsion shear strain gauges it can measure the 


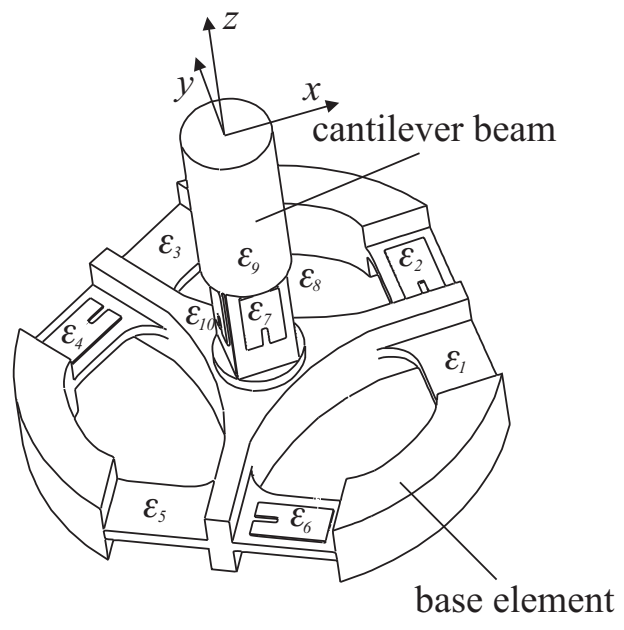

Figure 6. Mechanical structure of six dimensional force torque sensor.

$F_{x}, F_{y}$ and $M_{z}$.

$$
\begin{aligned}
F_{x} & =K_{1}\left(\epsilon_{7}-\epsilon_{9}\right) \\
F_{y} & =K_{2}\left(\epsilon_{8}-\epsilon_{10}\right) \\
F_{z} & =K_{3}\left[\left(\epsilon_{1}-\epsilon_{2}\right)+\left(\epsilon_{3}-\epsilon_{4}\right)+\left(\epsilon_{5}-\epsilon_{6}\right)\right] \\
M_{x} & =K_{4}\left(\epsilon_{3}-\epsilon_{4}-\epsilon_{5}+\epsilon_{6}\right) \\
M_{y} & =K_{5}\left[\epsilon_{1}-\epsilon_{2}-\frac{1}{2}\left(\epsilon_{3}-\epsilon_{4}+\epsilon_{5}-\epsilon_{6}\right)\right] \\
M_{z} & =K_{6}\left(\epsilon_{7}-\epsilon_{8}+\epsilon_{9}-\epsilon_{10}\right)
\end{aligned}
$$

Where $K_{i}$ are the calculation parameters.

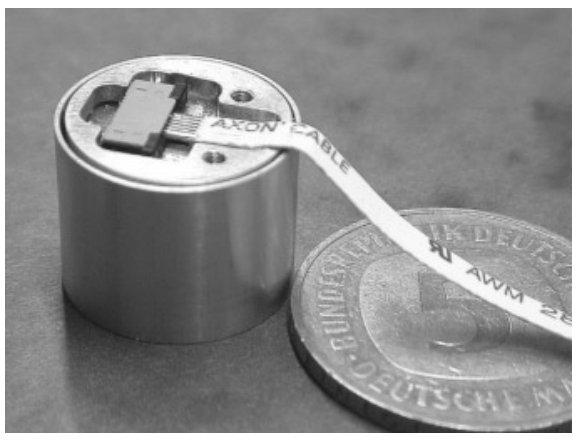

Figure 7. Six dimensional force torque sensor in fingertip.

Internal electronics: By using the latest technology the sensor with internal electronics can provide force and torque data at very high bandwidth and with very low noise. Signals from foil strain gage bridges are amplified and converted to digital representations of the force and torque applied to the sensor. All low level analog signals and the A/D converter (12bit) are within the sensor body, shielded from electromagnetic interference by the metal sensor body.

\subsection{Integrated Electronics}

One major goal of the design of the new DLR Hand was to fully integrate the electronics needed in the fingers and the palm in order to minimize weight, the amount of cables needed for a multisensory hand, and to increase the reliability by minimizing the amount of cables moved crossing the joints. In case of joints with a single degree of freedom we solved the problem of reliability by using flexible printed circuit boards (PCB) with appropriate bending space within the links (see top of Fig. 8). Tests showed after 100,000 cycles no

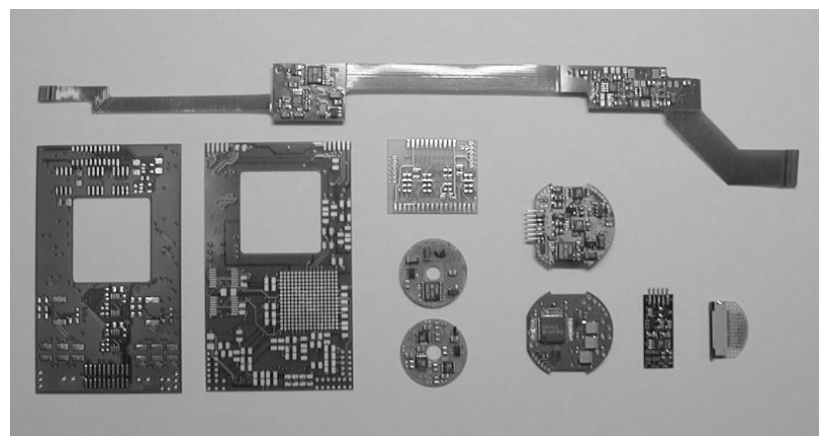

Figure 8. Set of PCBs in one finger.

visible or measurable effect on the flexible PCB.

In each link at least one serial ADC with 8 channels and 12 bit resolution converts the sensor signals as near as possible to the sensor circuitry into digital data. Thus only digital data is crossing any joint of the finger.

The power converters for driving the motors are located directly beside the motors and they are galvanically decoupled from the sensor electronics in order to minimize any noise induced by the running motors. Moreover the different fingers are galvanically decoupled from each other, too.

\subsection{Communication Architecture}

The control of the fingers and the hand is done by an external computer. In order to use the hand freely on different manipulators and to reduce cables and the possibility of noise in the sensor signals, we decided to design a fully integrated serial communication system. Each finger holds one communication controller in its base unit (see fig. 9). This controller is responsible for the collection and distribution of all information of interest. Furthermore it does some reasonable signal processing. 


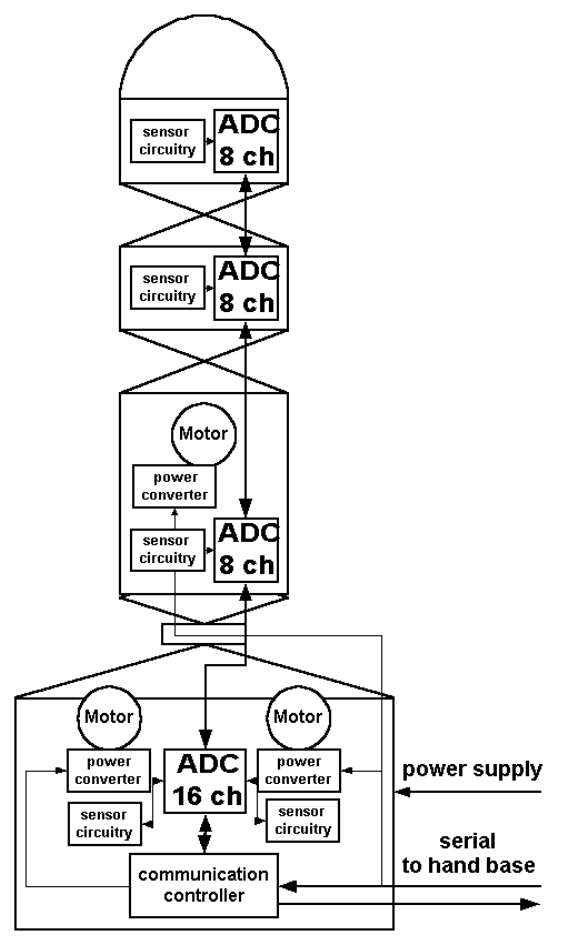

Figure 9. Electronics and communication in a finger.

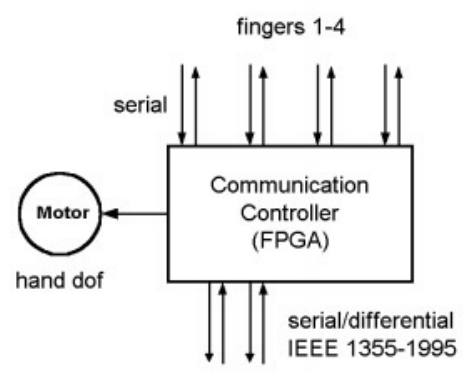

Figure 10. The communication controller in the hand base links the fingers to external computers.

It collects the data of all five ADCs per finger with together 40 channels of 12 bit resolution each and transmits these data to the communication controller in the hand base (see fig. 10). On the other hand it distributes the data from the control scheme to the actuators for finger control.

The communication controller in the hand base links the serial data stream of each finger to the data stream of the external control computer. By this hardware architecture we are able to limit the number of external cables of DLR's Hand II to a four line power supply and an eight line communication interface since the data is transmitted via differential lines. This inter- face even provides the possibility of using a quick-lock adaptor for autonomous tool exchange. Reducing external cabling from 400 (in Hand I) to 12 here, is one of the major steps forward in our new hand.

\section{Cartesian Impedance Control}

Salisbury [7] implemented cartesian stiffness control by using fingertip force sensors. The stiffness control scheme has the disadvantage of not being able to actively control the complete system dynamics, especially the system damping parameter. Hogan [2] introduced the impedance control scheme, which will improve system dynamic characteristics greatly. Using this control scheme we may control the complete impedance property of a finger:

$$
F_{\text {ext }}=M_{d} \ddot{x}_{k}+B_{d} \delta \dot{x}_{k}+K_{d} \delta x_{k}
$$

The equation above is central to the cartesian impedance control of the finger, where $M_{d}, B_{d}, K_{d}$ are the $3 \times 3$ diagonal desired target impedance parameters of the finger. The selection of parameters will determine the directions in which large impedance is desirable and the directions in which small impedance is desirable. $\delta x_{k}=x-x_{d}$ is a $3 \times 1$ vector of position errors, while $x, x_{d}$, and $F_{e x t}$ are $3 \times 1$ vectors of actual positions, desired positions and external forces in the fingertip coordinate system, respectively. An essential variable in the compliant motion is the generalized force, $F_{e x t}$, exerted by the end-effector. It is possible to select the coordinate system in view of the environment constrained in such a way that matrix $M_{d}, B_{d}$ and $K_{d}$ are diagonal. Each diagonal element is associated with a definite coordinate direction. For example, if a particular diagonal entry of the stiffness matrix $K_{d}$ is small, then the contact force in this direction is small. On the other hand, when a specific diagonal entry is chosen as a large number, indicating a stiff spring, even a small displacement in this direction results in a considerable force. A large stiffness coefficient requires an accurate position control. The desired environmental generalized force may be generated indirectly by controlling the corresponding positions accurately.

When a robot hand performs any fine manipulation, there is always need that the fingertip should be soft in the direction normal to the contact surface and hard tangential to the contact surface. Therefore the impedance should be adaptable to the orientation of the fingertip. Therefore, a cartesian impedance controller has been built as shown in Fig 11. In steady state, all measured and desired velocity and acceleration values are zero. This induces that the value of the 
steady state torque is the stiffness multiplied by the steady state deformation $\delta \theta$, and the fingertip behaves like a programmable spring.

\section{Conclusion and Future Work}

DLR's work on one side aims at the development of robonaut systems for space applications and on the other side at the terrestrial use of ultralight weight arms and multifinger hands on mobile platforms. The DLR Hand II, for us, is a big step forward in approaching the goal of hands with human-like size and performance. Currently one new hand is in use in our lab meanwhile the second hand is in the stage of assembly. Future work will be a redesign to get the hand ready for manufacturing in a small series und on the other hand to get the hand simplified and qualified for space applications. Parallel to this work various efforts towards autonomous grasping and manipulation with the new hand are in progress.

\section{References}

[1] Butterfaß, J.; Hirzinger, G.; Knoch, S.; Liu, H.: DLR's Multisensory Articulated Hand Part I: Hard-And Software Architecture, Proceedings of the IEEE Int. Conference on Robotics and Automation, Leuven, Belgium, 1998, pp. 2081-2086.

[2] Hogan N.: Impedance Control: An Approach to Manipulator: Part I-III, Trans. ASME J. Dyn. Syst., Meas., Contr., Vol. 107, 1985, pp. 1-24.

[3] Jacobsen, S.C.; Wood, J.E.; Knutti, D.F.; Biggers, K.B.: The UTAH/M.I.T. Dextrous Hand:
Work in Progress, The International Journal of Robotics Research, Vol. 3, No. 4, 1994.

[4] Liu, H.; Butterfaß, J.; Knoch, S.; Meusel, P.; Hirzinger, G.: A New Control Strategy for DLR's Multisensory Articulated Hand, Control Systems, Vol. 19, No. 2, April 1999, pp. 47-54.

[5] Lovchik, C.S.; Diftler, M.A.: The Robonaut Hand: A Dexterous Robot Hand for Space, Proceedings of the IEEE Int. Conference on Robotics and Automation, Detroit, 1999, S. 907-912.

[6] Reynaerts, D.: Control Methods and Actuation Technology for Whole-Hand Dextrous Manipulation, Dissertation, Katholieke Universiteit Leuven, 1995.

[7] Salisbury, J. K., Craig, J.J.: Articulated hands: force control and kinematics issues, The International Journal of Robotics Research, Vol. 1, 1982, pp. $4-17$.

[8] Townsend, W. T., Salisbury, J. K.: Mechanical Bandwidth as a Guideline to High-Performance Manipulator Design, Proceedings of the IEEE Int. Conference on Robotics and Automation, Scottsdale, USA, 1989, pp. 1390-1395.

[9] Yoshikawa, T.: Foundations of Robotics, Analysis and Control, The MIT Press, 1990.



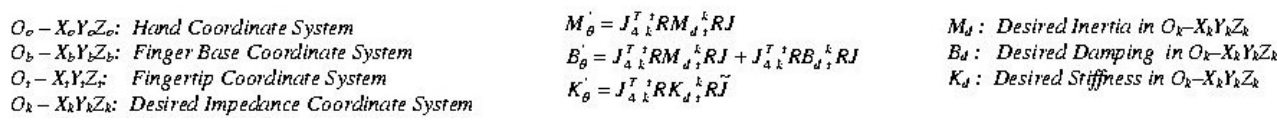

Figure 11. Block diagram of cartesian impedance control. 